\title{
Plant adaptogens increase lifespan and stress resistance in C. elegans
}

\author{
F. A. C. Wiegant $\cdot$ S. Surinova $\cdot$ E. Ytsma $\cdot$ \\ M. Langelaar-Makkinje · G. Wikman · J. A. Post
}

Received: 15 January 2008/Accepted: 20 May 2008/Published online: 7 June 2008

(C) The Author(s) 2008

\begin{abstract}
Extracts of plant adaptogens such as Eleutherococcus senticosus (or Acanthopanax senticosus) and Rhodiola rosea can increase stress resistance in several model systems. We now show that both extracts also increase the mean lifespan of the nematode $C$. elegans in a dose-dependent way. In at least four independent experiments, $250 \mu \mathrm{g} / \mathrm{ml}$ Eleutherococcus (SHE-3) and 10-25 $\mu \mathrm{g} / \mathrm{ml}$ Rhodiola (SHR-5) significantly increased life span between 10 and $20 \%(P<0.001)$, increased the maximum lifespan with 2-3 days and postponed the moment when the first individuals in a population die, suggesting a modulation of the ageing process. With higher concentrations, less effect was observed, whereas at the highest concentrations tested $(2500 \mu \mathrm{g} / \mathrm{ml}$ Eleutherococcus and $250 \mu \mathrm{g} / \mathrm{ml}$ Rhodiola) a lifespan shortening effect was observed of $15-25 \%(P<0.001)$. Both adaptogen extracts were also able to increase stress resistance in $C$. elegans: against a relatively short heat
\end{abstract}

F. A. C. Wiegant $(\bowtie) \cdot$ M. Langelaar-Makkinje ·

J. A. Post

Faculty of Science, Department of Biology, Cellular

Architecture \& Dynamics, Institute of Biomembranes,

Utrecht University, P.O. Box 80056, 3508 TB Utrecht,

The Netherlands

e-mail: f.a.c.wiegant@uu.nl

S. Surinova $\cdot$ E. Ytsma

University College Utrecht, Utrecht, The Netherlands

G. Wikman

Swedish Herbal Institute, Goteborg, Sweden shock $\left(35^{\circ} \mathrm{C}\right.$ during $\left.3 \mathrm{~h}\right)$ as well as chronic heat treatment at $26^{\circ} \mathrm{C}$. An increase against chronic oxidative stress conditions was observed in mev- 1 mutants, and during exposure of the wild type nematode to paraquat $(10 \mathrm{mM})$ or UV stress, be it less efficiently. Concerning the mode of action: both adaptogens induce translocation of the DAF-16 transcription factor from the cytoplasm into the nucleus, suggesting a reprogramming of transcriptional activities favoring the synthesis of proteins involved in stress resistance (such as the chaperone HSP-16) and longevity. Based on these observations, it is suggested that adaptogens are experienced as mild stressors at the lifespanenhancing concentrations and thereby induce increased stress resistance and a longer lifespan.

Keywords Adaptogens .

Eleutherococcus senticosus · Longevity ·

Rhodiola rosea $\cdot$ Stress resistance

\section{Introduction}

Extracts from plant adaptogens (such as Eleutherococcus senticosus and Rhodiola rosea) have been shown to increase the resistance of a variety of organisms against the damaging effect of different stress conditions (Wagner et al. 1994; Boon-Niermeijer et al. 2000; Davydov and Krikorian 2000; Brown et al. 2002; Khanum et al. 2005; Panossian and Wagner 2005). 
The terminology 'adaptogen' was originally used for various pharmacologically active compounds that could elicit a state of non-specifically raised resistance in mammals allowing them to counteract stressors and to adapt to exceptional strain (see Brekhman 1969, 1980; Meerson 1984). Later this term was also applied to a group of plants that exert adaptogenic properties (Brekhman 1969; Wagner et al. 1994). A review article by Brekhman (1969), one of the pioneers in plant adaptogen research, summarized almost 20 years of Russian research and made Western scientists aware of the research and the concepts. Indeed a large number of experiments indicated that physical performance and adaptive ability were increased when extracts from plants such as Eleutherococcus senticosus and Rhodiola rosea were applied to a variety of model systems (Wagner et al. 1994; Davydov and Krikorian 2000; Panossian and Wagner 2005 for reviews). In the definition of Brekhman (1980) adaptogens were described as having the following three characteristics: (1) an adaptogen must show a non-specific activity, i.e. an increase in power of resistance against physical, chemical or biological noxious agents; (2) it must have a normalizing influence independent of the nature of the pathological state; and (3) it must be harmless and must not influence normal body functions more than required.

In this way adaptogens are thought to reinforce the non-specific adaptive capacity to withstand situations of stress, to encourage a faster response towards stress conditions and to facilitate a more rapid return to normality (Wagner et al. 1994).

Since an increase in stress resistance has frequently been reported to coincide with an increase in lifespan in a variety of organisms (Lithgow et al. 1995; Johnson et al. 1996; Kapahi et al. 1999; Lithgow and Walker 2002; Muñoz and Riddle 2003), we wished to determine whether extracts of plant adaptogens could also trigger mechanisms that lead to a longer lifespan. For these studies, the nematode Caenorhabditis elegans was used, which has become a popular model to study aging and longevity, due to its short 3-week lifespan, easy culture conditions and rapid generation time (Guarente and Kenyon 2000).

In a recent paper, it was shown that one of the adaptogens (Rhodiola rosea) was able to increase the lifespan of Drosophila melanogaster (Jafari et al. 2007).
Although the mechanism in which plant adaptogens increase stress resistance is still a matter of debate, cell studies have shown an involvement of pathways leading to synthesis of one or more of the heat shock proteins as well as a development of oxidative stress resistance (Afanasiev et al. 1996; Chiu et al. 2003; De Sanctis et al. 2004; Wiegant et al. 2008). The IGF-1 signal transduction pathway has been demonstrated to play a crucial role in the regulation of both stress resistance and longevity in different model systems (Partridge and Gems 2002; Cypser et al. 2006; Houthoofd and Van Fleteren 2007; McElwee et al. 2007). Via this pathway the activity of specific transcription factors (such as the DAF-16/FOXO transcription factor) is influenced, which regulate the expression of proteins that are involved in metabolism, anti-oxidative defense, antimicrobial activity, detoxification and stress resistance (Lee et al. 2003; Murphy et al. 2003; Oh et al. 2006). The presence of DAF-16 was also shown to be essential for life extension of $C$. elegans by sub-lethal heat treatment (Cypser et al. 2006).

In the present paper, the question was raised whether standardized extracts of plant adaptogens are able to: (1) prolong the lifespan of the nematode Caenorhabditis elegans, as well as to increase their resistance to (heat) stress and if so (2) whether DAF-16 and HSP-16 are involved in the underlying mechanism.

\section{Materials and methods}

\section{Culturing of worms}

The nematode strains (N2, TJ356, CL2070 and Mev-1) were provided by the CGC (Caenorhabditis Genetics Center), which is funded by the NIH National Center for Research Resources (NCRR). Standard methods were used for strain maintenance, culturing and manipulating of worms (Brenner 1974; Stiernagle 1999). In particular, animals were cultured at $20^{\circ} \mathrm{C}$ on NGM agar plates seeded with live bacteria (E. coli, strain OP50) as food source.

\section{Strains}

The Bristol N2 wild-type strain was used for most lifespan assays as well as for stress resistance assays. The TJ356 strain carries an integrated DAF-16::GFP 
array in a wild-type background (Henderson and Johnson 2001) and was used to determine whether adaptogens were able to affect the intracellular localization of the daf 16 transcription factor (i.e. translocation between cytoplasm and nucleus). The CL2070 strain carries an integrated HSP-16::GFP array (Link et al. 1999) and was used to determine whether adaptogens were able to induce the synthesis of HSP16. The mev-l (kn1) mutant strain has a defect in the succinate dehydrogenase cytochrome $b$ large subunit in complex II of the mitochondrial electron transport chain and shows hypersensitivity to oxygen and paraquat (Ishii et al. 1998). An elevated level of reactive oxygen species (ROS) in mev-1 mutants has been reported. This latter strain was used to determine whether extracts from adaptogens may have ROS (superoxide) quenching activities.

Lifespan and survival assays

For assaying lifespan, age-synchronous N2 worms were prepared by the alkaline hypochlorite method (Sulston and Brenner 1974) and isolated eggs were placed on NGM agar plates to hatch. Two day-old synchronized animals were subsequently placed on NGM agar plates with OP50 as food source and $50 \mu \mathrm{M}$ 5-fluorodeoxyuridine (FUdR was obtained from Sigma) which was used to block progeny development (Hosono 1978). In case the agar was supplemented with the plant adaptogen, the extract was added during the preparatory process of the agar plates. Each day the dishes were evaluated for survival and animals were scored dead when they did not move after repeated taps with a pick. Experiments were usually performed 3 times (unless indicated otherwise). Per experiment, a minimum of 100 worms divided over 3-4 dishes, was used to evaluate the effect of each single concentration of adaptogen.

The concentrations of adaptogens used in lifespan experiments did not affect the growth of the OP50 bacteria as indicated by visual inspection of the bacterial mass on top of the agar plates, which remained the same or even slightly increased. In addition, the effect of the adaptogens on bacterial growth was studied in liquid cultures of the OP50 bacteria using Optical Density measurements of the bacteria grown in the presence or absence of different concentrations of plant adaptogens. Again no effect on bacterial growth characteristics was observed (not shown).
In stress resistance assays, the number of surviving animals was monitored for several days following exposure to the indicated stressor (heat shock at $35^{\circ} \mathrm{C}$ during $180 \mathrm{~min}$; exposure to paraquat (Sigma) for $24 \mathrm{~h}$; and an exposure to UV, which was performed in a UV Stratalinker 1800 (Stratagene) with a dose of $1000 \mathrm{~J} / \mathrm{m}^{2}$. The effect of the stress conditions on survival in the presence or absence of adaptogens was further compared with survival values in control cultures.

Statistical analysis

Results of lifespan experiments were analyzed using Kaplan-Meier survival analysis and compared among groups scoring for significance using the log-rank test. The results of survival values following stress conditions were analyzed using Student $t$-test. For Table 1, the average of the mean lifespan, the minimum and the maximum lifespan of a set of independent experiments were calculated and expressed as mean \pm SD. SPSS was used as statistical software. A $P$-value of 0.05 or less was considered to be statistically significant.

\section{Adaptogen extracts}

Extracts from plant adaptogens (in powder form) were supplied by the Swedish Herbal Institute. The extract of roots from Eleutherococcus senticosus (Rupr. et Maxim) Harms (SHE-3) was containing 2.1\% Eleutherosides $(\mathrm{E}+\mathrm{B})$, whereas the extract from roots of Rhodiola rosea L (SHR-5) was containing 3.0\% salidroside. The powder was dissolved in sterile water and stored as a stock concentration of $100 \mathrm{mg} / \mathrm{ml}$ at $4^{\circ} \mathrm{C}$ until use.

Visualization of DAF-16 localization with fluorescence microscopy

In order to examine the intracellular distribution of DAF-16 in the living nematode, the TJ356 strain was used (Henderson and Johnson 2001). In this strain, the gene coding for Green Fluorescent Protein (GFP) was fused to the daf-16 gene such that GFP becomes attached to the last predictable residue of the DAF-16 protein. In this way GFP is used as a reporter of DAF-16 localization (Henderson and Johnson 2001). In control conditions the transcription factor DAF-16 is found in the cytoplasm. However, when the organism is subjected to mild thermal stress DAF-16 is translocated 
Table 1 Adaptogens added early in life

\begin{tabular}{|c|c|c|c|c|c|c|c|c|c|}
\hline & \multicolumn{2}{|c|}{ Lifespan (in days) } & \multirow[t]{2}{*}{$n$} & \multicolumn{2}{|c|}{ First and last death } & \multirow[t]{2}{*}{ Total no. of worms } & \multicolumn{2}{|c|}{ Lifespan (\% of control) } & \multirow[t]{2}{*}{$P$-Value } \\
\hline & Mean & SD & & Min & Max & & Mean & SEM & \\
\hline \multicolumn{10}{|c|}{ Eleutherococcus (SHE-3) $(\mu \mathrm{g} / \mathrm{ml})$} \\
\hline Control & 14.31 & 1.05 & 7 & 8.3 & 20.8 & 806 & 100.00 & 2.50 & \\
\hline Eleu-100 & 15.13 & 0.88 & 4 & 8.8 & 21.3 & 484 & 105.34 & 2.68 & $P=0.036$ \\
\hline Eleu-250 & 16.57 & 1.28 & 6 & 10.2 & 23.3 & 731 & 115.79 & 1.20 & $P<0.001$ \\
\hline Eleu-500 & 15.43 & 0.82 & 3 & 9.0 & 22.3 & 359 & 107.46 & 2.42 & $P=0.005$ \\
\hline Eleu-1000 & 13.46 & 1.01 & 4 & 8.3 & 19.8 & 368 & 93.79 & 3.56 & ns \\
\hline Eleu-2500 & 10.65 & 1.15 & 1 & 7.0 & 18.0 & 60 & 77.06 & 5.00 & $P<0.001^{*}$ \\
\hline \multicolumn{10}{|c|}{ Rhodiola (SHR-5) ( $\mu \mathrm{g} / \mathrm{ml})$} \\
\hline Control & 14.85 & 1.01 & 6 & 8.2 & 22.5 & 1146 & 100.00 & 2.50 & \\
\hline Rho-2.5 & 15.51 & 1.50 & 1 & 8.0 & 21.0 & 53 & 101.91 & 2.50 & ns* \\
\hline Rho-10 & 17.14 & 1.50 & 4 & 9.5 & 25.3 & 508 & 115.14 & 1.86 & $P<0.001$ \\
\hline Rho-25 & 16.60 & 1.15 & 5 & 8.8 & 24.4 & 709 & 110.77 & 0.99 & $P<0.001$ \\
\hline Rho-50 & 15.82 & 0.74 & 3 & 8.0 & 24.3 & 394 & 103.76 & 1.86 & $P=0.032$ \\
\hline Rho-100 & 14.57 & 0.52 & 3 & 8.0 & 20.3 & 325 & 96.70 & 2.27 & ns \\
\hline Rho-250 & 13.54 & 1.50 & 1 & 7.0 & 19.0 & 187 & 86.02 & 2.50 & $P<0.001^{*}$ \\
\hline
\end{tabular}

Lifespan of $C$. elegans in the presence of different concentrations of adaptogens. The average of the mean lifespan ( \pm Standard Deviation) of a set of independent experiments $(n)$ is shown. Min: indicates the moment (day) that the first dead worm was observed in the experiments. Max: indicates the maximum lifespan observed per indicated concentration. Total number of worms used per condition in independent experiments is shown. The normalized lifespan is shown as percentage of control of independent experiments and $P$-values express significance using independent $t$-Test (* $P$-value from Kaplan-Meier survival analysis, log-rank test in case data are from 1 experiment)

to the nucleus. The effect of different concentrations of adaptogens (100, 250, 500, $1000 \mu \mathrm{g} / \mathrm{ml}$ Eleutherococcus and 10, 25, 50, 100 and $250 \mu \mathrm{g} / \mathrm{ml}$ Rhodiola)(SHR-5) was studied and compared with the effect of a mild heat shock at $35^{\circ} \mathrm{C}$ during 20 min which was used as a positive control. DAF-16 localization was examined in approximately 20 animals per condition that were mounted in $2 \%$ agarose pads. Nematodes were examined with a Leitz orthoplan fluorescence microscope using $25 \times$ and $40 \times$ objectives. Images were acquired with a digital camera.

Detection of HSP-16 induction with Gel electrophoresis, Western Blotting \& Immunolabeling

Standard procedures were used to obtain protein samples for each set of conditions by collecting worms from plates by washing with a buffer and subsequent centrifugation. Pellets were boiled at $100^{\circ} \mathrm{C}$ for $15 \mathrm{~min}$ in sample buffer and stored at $-20^{\circ} \mathrm{C}$ until electrophoresis. The same amount of protein was loaded on gels. Gel electrophoresis, blotting and detection of specific proteins were performed with standard procedures. Blots were equilibrated in washing solution and incubated with a monoclonal primary antibody (mouse $\alpha$-GFP (Clontech, USA)) for $1 \mathrm{~h}$. The antibody-antigen complex was rinsed with washing solution and the membranes were incubated with secondary polyclonal antibody (donkey anti mouse coupled to peroxidase (Jackson Immuno Research, USA)) for $45 \mathrm{~min}$. After washing, the presence of HSP-16 was detected using an enhanced chemiluminescence detection system according to the manufacturer (PerkinElmer Life Sciences), using either normal or hypersensitive film.

\section{Results}

Do plant adaptogens prolong lifespan?

In order to determine whether plant adaptogens are able to enhance longevity, populations of C. elegans 
were exposed to different concentrations of standardized extracts from Eleutherococcus senticosus (SHE-3) or Rhodiola rosea (SHR-5). A concentration dependent modulation of lifespan was observed. We were able to replicate these results in at least four independent assays. Representative survival curves of the effect of Eleutherococcus and of Rhodiola are shown in Figs. 1 and 2 respectively. In general, low concentrations hardly extended the normal mean lifespan of about 14.5 days (Table 1), whereas higher concentrations significantly extended the mean lifespan with 2 days or more, which is an extension of the mean lifespan of about $15 \%$. The highest concentrations had an adverse effect and even shortened lifespan significantly (Table 1).

The most effective beneficial concentrations of Eleutherococcus $(250 \mu \mathrm{g} / \mathrm{ml})$ and of Rhodiola $(10 \mu \mathrm{g} / \mathrm{ml})$ did not only extend the mean lifespan,

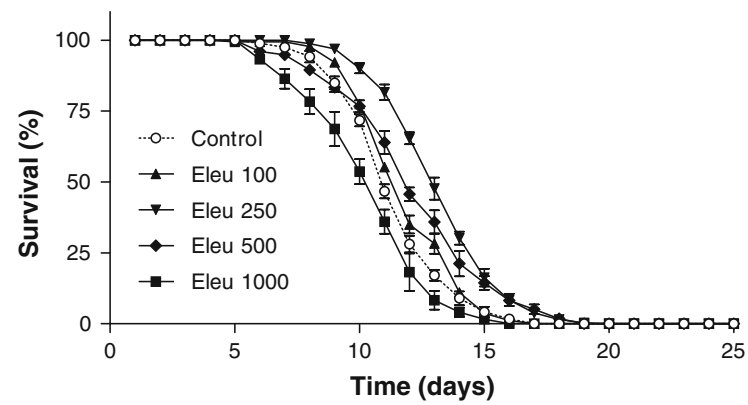

Fig. 1 Eleutherococcus senticosus extract (SHE-3) causes a concentration-dependant increase in lifespan of N2 wild-type C. elegans. A highly significant change in longevity is observed when Kaplan-Meier survival analysis (log-rank test) is used to compare among groups $(P<0.001)$. Significance per concentration $(\mu \mathrm{g} / \mathrm{ml})$ is shown in Table 1

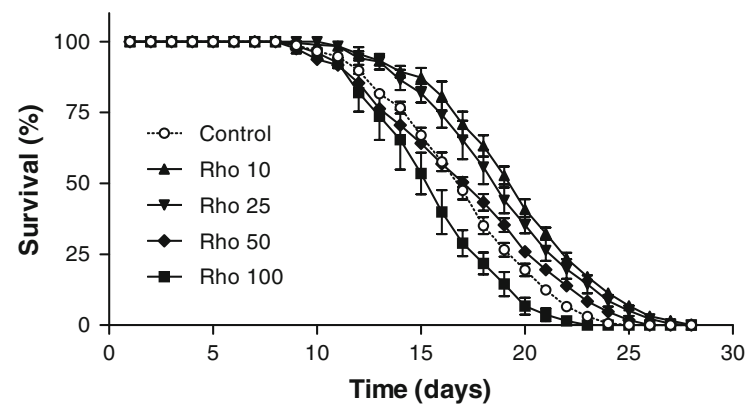

Fig. 2 Incubation of C. elegans in different concentrations $(\mu \mathrm{g} / \mathrm{ml})$ of Rhodiola rosea extract (SHR-5) results in a highly significant modulation of lifespan $(P<0.001)$. Kaplan-Meier survival analysis (log-rank test) is used to compare longevity among the indicated groups but were also able to increase the maximum lifespan with 2.5-3 days (Table 1). In addition, the day that the first dead worms were observed in the survival assays was also postponed at these concentrations (from 8.3 to 10.2 for Eleutherococcus and from 8.2 to 9.5 for Rhodiola).

With respect to Eleutherococcus, a concentration of $100 \mu \mathrm{g} / \mathrm{ml}$ only showed a modest increase in the mean lifespan, whereas $250 \mu \mathrm{g} / \mathrm{ml}$ showed a clear and highly significant lifespan enhancing effect $(P<0.001$ : Kaplan-Meier survival analysis with log-rank test). The increase in mean lifespan was from 14.3 days in control cultures to 16.6 days in cultures exposed to a concentration of $250 \mu \mathrm{g} / \mathrm{ml}$ which represents a mean increase in lifespan of almost $16 \%$ (Table 1). In each of four independent experiments, the difference in lifespan of all treated groups in comparison with control values was highly significant $(P<0.001)$. Moreover, the effect of $250 \mu \mathrm{g} / \mathrm{ml}$ Eleutherococcus showed a consistent and highly significant increase in lifespan $(P<0.001)$. Higher concentrations (such as $500 \mu \mathrm{g} / \mathrm{ml}$ ) showed to be less effective than some of the lower concentrations, whereas at the highest concentrations tested (1000 and $2500 \mu \mathrm{g} / \mathrm{ml})$ a negative effect was observed at which the mean lifespan was reduced in comparison with control cultures, which was significant for the highest concentration $(2500 \mu \mathrm{g} / \mathrm{ml}$; $P<0.001)$.

With respect to Rhodiola, a very low concentration, such as $2.5 \mu \mathrm{g} / \mathrm{ml}$ did not influence lifespan markedly (Table 1), whereas a concentration of 10 and $25 \mu \mathrm{g} / \mathrm{ml}$ were the most effective ones in extending the mean lifespan with 2 days or more, which represents an increase of $10-15 \%(P<0.001)$. As shown in Fig. 2, higher concentrations of Rhodiola $(50 \mu \mathrm{g} / \mathrm{ml})$ did not markedly influence the mean lifespan whereas the highest concentrations tested $(100 \mu \mathrm{g} / \mathrm{ml})$ even shortened the mean lifespan significantly $(P<0.001$; Fig. 2$)$. A concentration of $250 \mu \mathrm{g} / \mathrm{ml}$ shortened the mean lifespan to about $86 \%$ of control values $(P<0.001$; Table 1$)$.

The beneficial lifespan-enhancing effect at low concentrations of adaptogens and an adverse effect at higher concentrations is clearly observed in Figs. 3 and 4. The data obtained in a set of independent experiments was used to illustrate the concentrationdependent modulation of the mean lifespan in C. elegans. Interestingly, the optimum effective 


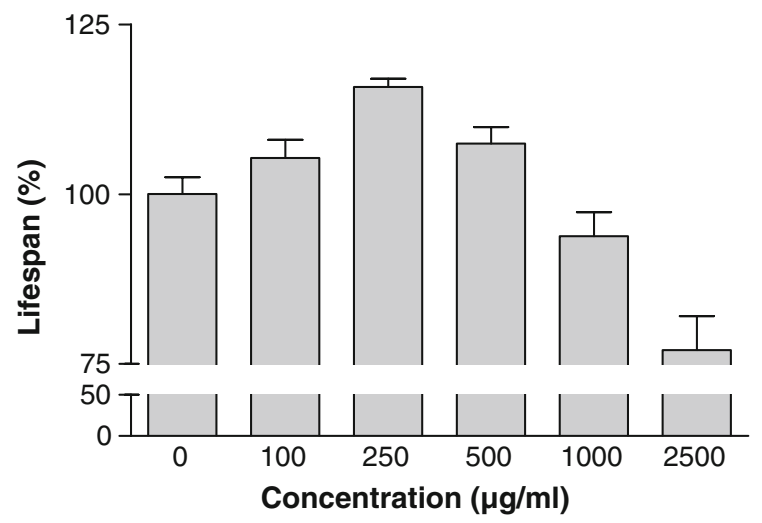

Fig. 3 Effect of Eleutherococcus senticosus extract (SHE-3) on the mean lifespan of $C$. elegans as percentage of the control value $(100 \%)$. Results are the average of four independent experiments in which a total of 2808 worms were used. Significance of the indicated concentrations is shown in Table 1

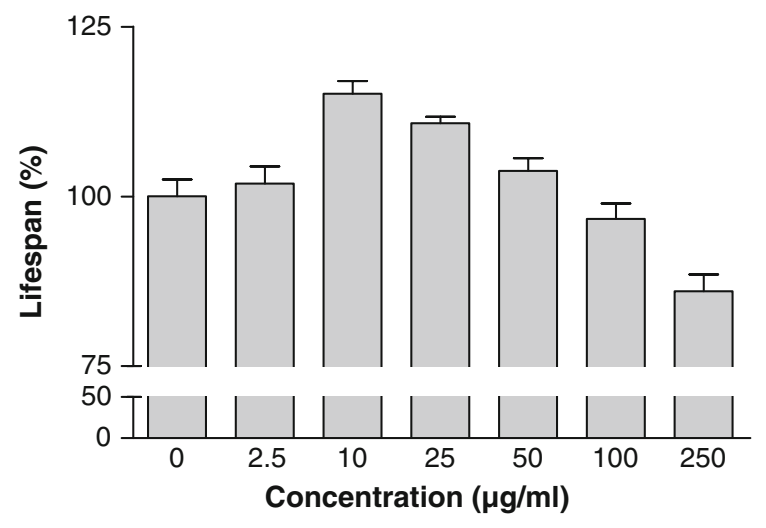

Fig. 4 Effect of Rhodiola rosea extract (SHR-5) on the mean lifespan of $C$. elegans as percentage of the control value. The average is shown of four independent experiments in which a total of 3322 worms were used. Significance of the effect in comparison with control is shown in Table 1 for each indicated concentration

concentration of Rhodiola is about 10-fold lower than the effective concentration of Eleutherococcus.

Are plant adaptogens still effective when added later in life?

Since the extracts were present during most of the lifespan of the worms (starting at day 2 until the end), it was interesting to inquire whether the extracts are still effective when added just before the moment that the first worms start to die (which usually occurs around day 8) which roughly corresponds to about

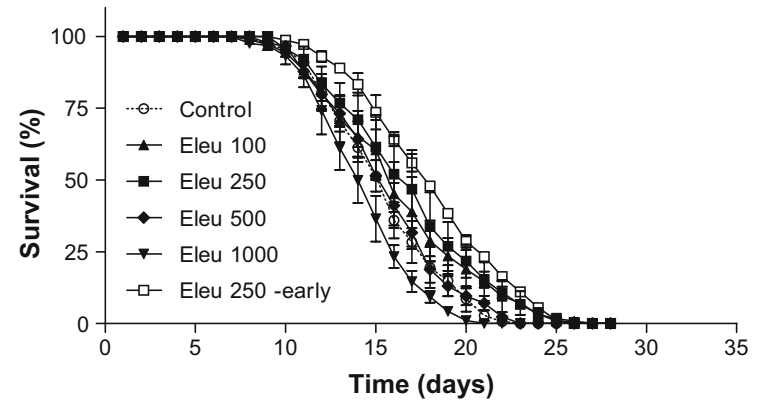

Fig. 5 Effect of different concentration of Eleutherococcus senticosus extract (SHE-3; 100, 250, 500 or $1000 \mu \mathrm{g} / \mathrm{ml}$ ) on longevity of $C$. elegans when added later in life (at an age of 7 days) in comparison with non-treated individuals (open circles) or with individuals that received the extract $(250 \mu \mathrm{g} /$ $\mathrm{ml}$ ) already at an age of 2 days (open squares). A total of 837 worms were used. Eleutherococcus (100 and $250 \mu \mathrm{g} / \mathrm{ml})$ increased the mean lifespan of 15.53 days in control cultures significantly with $7.9 \%$ and $11.7 \%$ respectively $(P<0.001$ for both concentrations), but not as much as when Eleutherococcus $(250 \mu \mathrm{g} / \mathrm{ml})$ was added early in life (increase of mean lifespan with $17.8 \%(P<0.001)$. The highest concentration $(1000 \mu \mathrm{g} /$ $\mathrm{ml})$ produced a life-shortening effect of $6.4 \%(P<0.001)$

$50 \%$ of the mean lifespan. In Fig. 5, it can be observed that Eleutherococcus (100 and $250 \mu \mathrm{g} / \mathrm{ml}$ ) was able to extend the mean lifespan significantly (from 15.53 days in control condition to 16.76 days $(100 \mu \mathrm{g} / \mathrm{ml}, P<0.001)$ and to a value of 17.35 days $(250 \mu \mathrm{g} / \mathrm{ml}, P<0.001)$. Although this value of $11.7 \%$ increase in mean lifespan is less in comparison with the adaptogen added early in life $(17.8 \%$ in this specific experiment), it is still highly significant. The highest concentration $(1000 \mu \mathrm{g} / \mathrm{ml})$ was again found to have a life-shortening effect to $93.6 \%$ of the control value $(P<0.001)$. Rhodiola $(10$ and $25 \mu \mathrm{g} / \mathrm{ml})$ was found to extend the mean lifespan with about 1.5 days when added later in life $(P<0.001$; Fig. 6).

Do plant adaptogens enhance stress resistance?

One of the characteristics of Eleutherococcus and of Rhodiola is that they have been shown to increase the resistance against different stress conditions in a variety of model systems (Boon-Niermeijer et al. 2000; Brown et al. 2002; Davydov and Krikorian 2000; Panossian and Wagner 2005). Therefore, we addressed the question whether adaptogen extracts are also able to enhance resistance against stress in C. elegans. Concentrations of adaptogens were chosen that showed a strong effect with respect to lifespan 


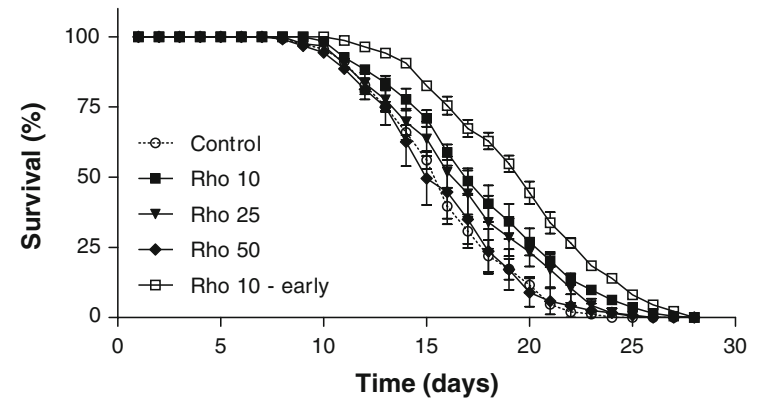

Fig. 6 Effect of different concentrations of Rhodiola rosea extract (SHR-5; 10, 25 or $50 \mu \mathrm{g} / \mathrm{ml}$ ) on longevity of C. elegans when added later in life (at an age of 7 days) in comparison with non-treated individuals (open circles) or with individuals that received the extract $(10 \mu \mathrm{g} / \mathrm{ml})$ already at an age of 2 days (open squares). A total of 616 individuals were used. Rhodiola (10 and $25 \mu \mathrm{g} / \mathrm{ml}$ ) increased the mean lifespan of 15.86 days in control cultures significantly with $11.5 \%$ and $8.0 \%$ respectively $(P<0.001$ for both concentrations). The highest concentration $(100 \mu \mathrm{g} / \mathrm{ml})$ did not affect the mean lifespan in comparison with control

prolongation $(250 \mu \mathrm{g} / \mathrm{ml}$ Eleutherococcus and $25 \mu \mathrm{g} /$ $\mathrm{ml}$ Rhodiola). Stress conditions were selected (heat shock and UV exposure) that would kill about $50 \%$ of the population in the following 4-5 days after exposure to the stress condition. In this respect, a heat shock at $35^{\circ} \mathrm{C}$ during $3 \mathrm{~h}$, or exposure to UV (at a dose of $1000 \mathrm{~J} / \mathrm{m}^{2}$ ) did fit the purpose. Worms were put on agar plates in which the indicated concentration of adaptogen extract was present for a period of 4 days, before they were exposed as adults (at the age of 6 days) to the above mentioned stress conditions. During the subsequent days, the number of surviving worms was evaluated daily. In Fig. 7a, the survival

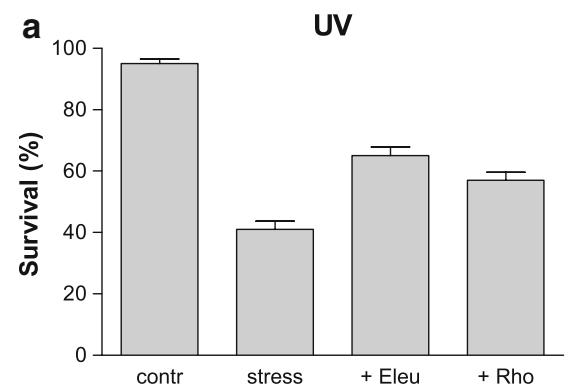

Fig. 7 Adaptogens (Eleutherococcus senticosus $(250 \mu \mathrm{g} / \mathrm{ml})$ and Rhodiola rosea (SHR-5; $25 \mu \mathrm{g} / \mathrm{ml}$ )) increase stress resistance of $C$. elegans against UV $\left(1000 \mathrm{~J} / \mathrm{m}^{2}\right)$ or against heat shock $\left(35^{\circ} \mathrm{C}\right.$ during $\left.3 \mathrm{~h}\right)$. Cultures of C. elegans were incubated with the adaptogen during 3 days before exposure to percentage is shown 3 days after UV illumination. In the presence of adaptogens worms showed higher survival values. Without any adaptogens, only $40.6 \%$ of the worms were alive, whereas this percentage was increased to $65.3 \%$ in the presence of Eleutherococcus and to $56.1 \%$ in the presence of Rhodiola, which is highly significant $(P<0.005)$ in both cases.

When cultures of $C$. elegans were exposed to a heat shock at $35^{\circ} \mathrm{C}$ for a time period of $3 \mathrm{~h}$, the surviving control population was reduced to about $56.8 \%$ as was measured 4 days after the heat shock (Fig. 7b). However, in the presence of $250 \mu \mathrm{g} / \mathrm{ml}$ Eleutherococcus or $25 \mu \mathrm{g} / \mathrm{ml}$ Rhodiola, this percentage was increased to $69.4 \%$ and $74.0 \%$ respectively (both $P<0.005$ ), indicating a better ability to cope with heat stress leading to higher survival percentages due to the presence of extracts of plant adaptogens before and during stress.

In addition, the effect of adaptogens was also determined on the mean lifespan in survival curves following the indicated stress condition (Figs. 8 and 9). Exposure to UV (at a dose of $1000 \mathrm{~J} / \mathrm{m}^{2}$ ) reduced the normal mean lifespan of 14.6 days to 9.6 days (Fig. 8). However, when the worms were incubated in Eleutherococcus $(250 \mu \mathrm{g} / \mathrm{ml})$ before and during UV illumination the mean lifespan was reduced to only 11.1 days instead of 9.6 days, which represents a significant protection $(P<0.001)$.

In Fig. 9 it can be observed that a heat shock at $35^{\circ} \mathrm{C}$ for $3 \mathrm{~h}$ caused a larger decrease in survival values during the first week following heat stress in comparison with the control (non heat-shocked) population. However, the slope of the curve indicating the rate at which the population size decreased is

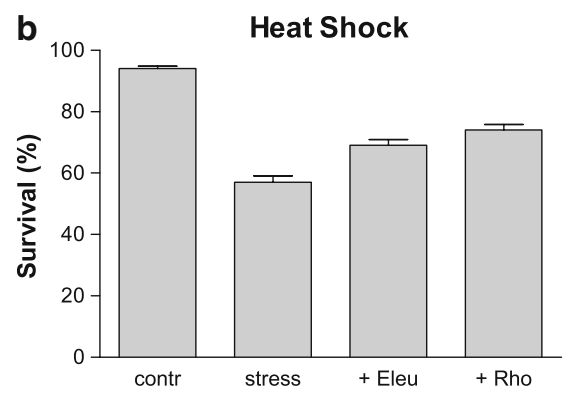

the indicated stress condition. The percentage of surviving worms was evaluated 3 days after exposure to UV (a) or heat shock (b). In all cases the survival percentage was significantly higher in comparison with survival values in control (nonadaptogen treated but stressed) cultures $(P<0.005)$ 


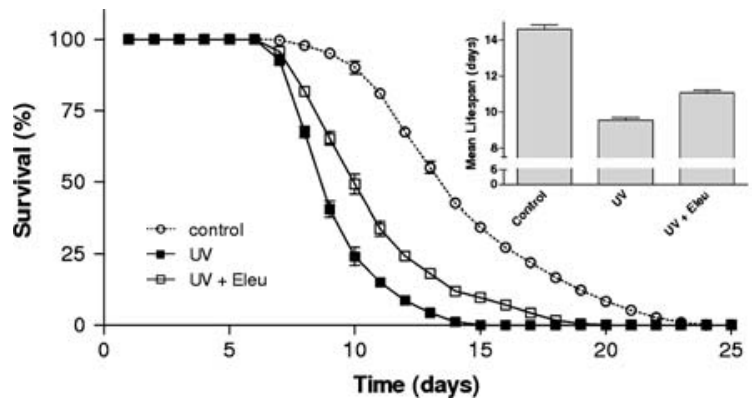

Fig. 8 The protective effect of Eleutherococcus senticosus $(250 \mu \mathrm{g} / \mathrm{ml})$ is shown on lifespan in C. elegans following exposure to $\mathrm{UV}\left(1000 \mathrm{~J} / \mathrm{m}^{2}\right)$. Incubation of cultures of C. elegans with the adaptogen started 3 days before exposure to UV and survival was monitored at regular intervals. The mean lifespan in control cultures of 14.6 days (open circles) is reduced to 9.6 days (closed squares) due to UV exposure, whereas in the presence of the adaptogen the mean lifespan is reduced to only 11.1 days (open squares), being a significant protection $(P<0.001)$

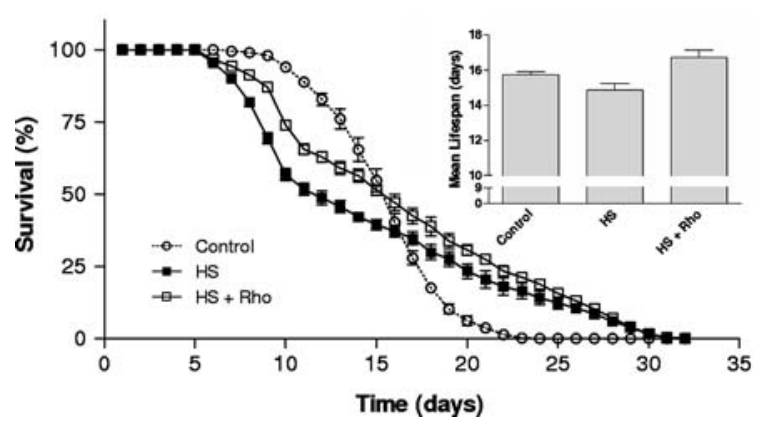

Fig. 9 The protective effect of Rhodiola (SHR-5; $25 \mu \mathrm{g} / \mathrm{ml}$ ) is shown on lifespan of $C$. elegans following exposure to heat shock $\left(35^{\circ} \mathrm{C}\right.$ during $\left.3 \mathrm{~h}\right)$. Incubation of cultures of $C$. elegans with the adaptogen started 3 days before heat shock and survival was subsequently monitored at regular intervals. The mean lifespan following heat shock of 14.85 days (closed squares) is increased to 16.72 days (open squares) in the presence of the adaptogen, which is a significant protection $(P<0.001)$. Open circles represent survival of control (nonstressed and non-adaptogen treated) cultures of $C$. elegans

much smaller than the slope of the curve of the control culture. Apparently there is a subset of individuals in the heat stressed population that far outlived the individuals from the control population. The maximum lifespan is increased from 24 days in control to 32 days in the heat stressed culture. A number of worms therefore benefited from the heat shock in the sense that they lived much longer than usual. The presence of Rhodiola $(25 \mu \mathrm{g} / \mathrm{ml})$ during heat shock prevented the rapid decrease in survival values during the first week after heat shock (Fig. 9), which lead to a significant protection and an increase in mean lifespan in comparison with the heat shock condition alone (16.72 days vs. 14.85 days in the heat shock condition $(P<0.001))$. However, the slope in which the survival curve gradually decreases was higher in the worm population that was heat stressed in the presence of Rhodiola in comparison with the control heat stressed population, finally leading to a similar maximum lifespan of 32 days.

The effect of adaptogens was also tested in the presence of a chronic stress condition, such as incubation at a higher temperature $\left(26^{\circ} \mathrm{C}\right)$ or in the presence of paraquat $(10 \mathrm{mM})$, the latter being used as an intracellular free-radical-generating compound. Although both adaptogens offered protection, Eleutherococcus $(250 \mu \mathrm{g} / \mathrm{ml})$ provided a better protection during these chronic stress conditions in comparison with Rhodiola ( $25 \mu \mathrm{g} / \mathrm{ml}$; Fig. 10). The population of surviving worms was reduced to $11.1 \%$ after 4 days at $26^{\circ} \mathrm{C}$. In the presence of Eleutherococcus, $20.9 \%$ stayed alive $(P=0.001)$, whereas $16.6 \%$ managed to survive in the presence of Rhodiola $(P=0.015)$.

When worms were continuously exposed to paraquat (10 $\mathrm{mM}$ ) for 4 days, $23.5 \%$ of the initial population was still alive. When paraquat exposure was accompanied with Eleutherococcus $(250 \mu \mathrm{g} / \mathrm{ml}), 29.9 \%$ of the initial population survived $(P=0.029)$ whereas $24.3 \%$ stayed alive in the presence of Rhodiola, which was not significantly different in comparison with exposure to paraquat alone $(P>0.05$; Fig. 10$)$.

To further examine whether adaptogens were able to protect against chronic oxidative stress, mev-1 animals were used, which have a mutation in the cytochrome $b$ large subunit of mitochondrial complex II (Ishii et al. 1998). This mutation results in overproduction of superoxide and increased oxidative stress along with accelerated ageing and reduced lifespan. As is shown in Fig. 11, Eleutherococcus $(250 \mu \mathrm{g} / \mathrm{ml})$ was able to increase the mean lifespan from 9.36 days in control cultures to 10.22 days $(P=0.003)$, an increase of $9.2 \%$. However, this increase was not as pronounced as the effect of Eleutherococcus in the wild type C. elegans. Rhodiola $(25 \mu \mathrm{g} / \mathrm{ml})$ did not have a significant effect in mev-1 animals. The mean lifespan is only slightly increased to 9.66 days (ns). Higher concentrations of both adaptogens resulted in a reduction of mean lifespan (not shown). 


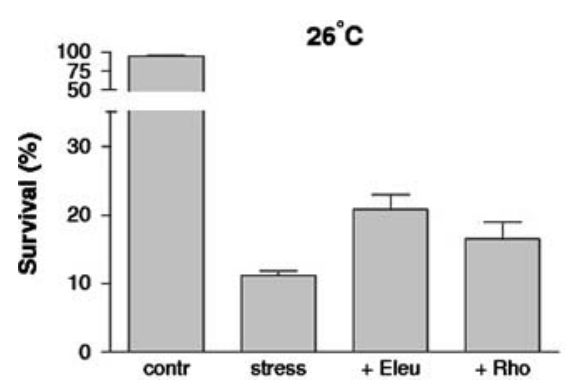

Fig. 10 Adaptogens increase stress resistance of C. elegans during chronic stress conditions. Exposure of C. elegans to $26^{\circ} \mathrm{C}$ during 4 days reduces survival values to $11.1 \%$, whereas in the presence of Eleutherococcus $(250 \mu \mathrm{g} / \mathrm{ml})$ the chronic elevated temperature reduces survival values to $20.9 \%(P=0.001)$ and in the presence of Rhodiola (SHR-5; $25 \mu \mathrm{g} / \mathrm{ml}$ ) survival

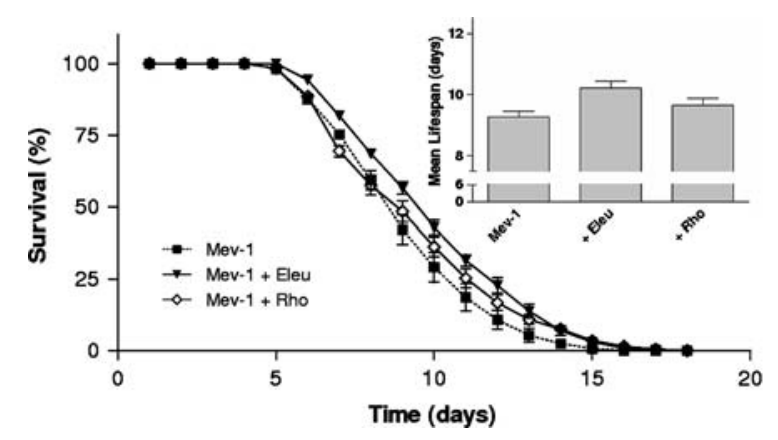

Fig. 11 Effect of adaptogens (Eleutherococcus senticosus $(250 \mu \mathrm{g} / \mathrm{ml})$ and Rhodiola rosea (SHR-5; $25 \mu \mathrm{g} / \mathrm{ml})$ ) on lifespan of the mutant C. elegans strain mev-1. Eleutherococcus increased the mean lifespan significantly from 9.36 days (control: closed squares) to 10.22 days (closed triangles; $P=0.003)$. Rhodiola did not significantly modulate the lifespan at the studied concentration

Do plant adaptogens affect the cellular localization of DAF16?

After demonstrating that plant adaptogens are able to both enhance lifespan as well as to increase the resistance against a number of stress conditions, it was inquired how the extracts of Eleutherococcus or of Rhodiola may manage to have these effects. Since the IGF-1 pathway and especially the DAF-16 transcription factor is known to play a crucial role in longevity, ageing and stress resistance (Partridge and Gems 2002; Lee et al. 2003; Murphy et al. 2003; Oh et al. 2006; Cypser et al. 2006), we studied whether the extracts were able to affect the cellular localization of DAF-16. In the nucleus, DAF-16 is known to activate transcription of a large number of

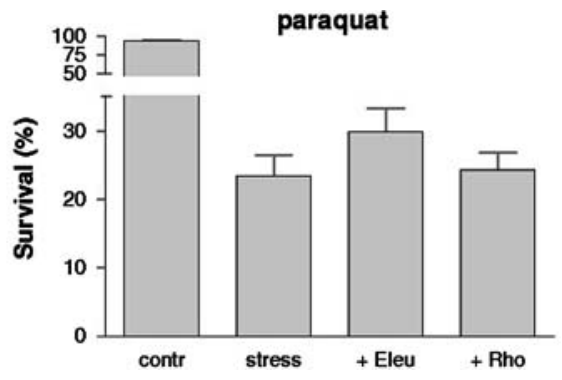

is $16.6 \%$ which also represents a significant protection $(P=0.015)$. Continuous exposure of $C$. elegans to paraquat $(10 \mathrm{mM})$ for 4 days reduces the survival of control cultures to $23.5 \%$. Only Eleutherococcus $(250 \mu \mathrm{g} / \mathrm{ml})$, but not Rhodiola, was able to significantly improve oxidative stress resistance to a survival of $29.9 \%(P=0.029)$

genes that increase stress resistance and that lead to longevity. It was thus postulated that this transcription factor is involved in the mechanism of action of plant adaptogens. To explore this hypothesis, the transgene strain TJ356 was used in which DAF-16 was coupled to GFP and in which the cellular localization of the transcription factor could thus be observed in living animals using fluorescence microscopy (Henderson and Johnson 2001).

Initially, the localization of DAF-16 was studied in control conditions and in cultures that were exposed to a heat shock. As shown in Fig. 12, a diffuse homogenous distribution was observed in worms under control conditions. Henderson and Johnson (2001) found that DAF-16 rapidly translocates from the cytoplasm to the nucleus when the worms are exposed to environmental stressors such as heat, oxidative stress and starvation. Following exposure to a short heat shock ( $35^{\circ}$ for $20 \mathrm{~min}$ ), a clear nuclear localization of DAF-16 was indeed detected (Fig. 12). A similar effect was observed following exposure to paraquat $(50 \mathrm{mM}$ for $1 \mathrm{~h}$ ), as well as after food restriction ( $24 \mathrm{~h}$ in the absence of any bacteria to feed on; not shown). In further experiments, the heat shock $\left(35^{\circ} \mathrm{C}\right.$ for $\left.20 \mathrm{~min}\right)$ was used as a positive control.

Subsequently, the question was asked whether extracts from plant adaptogens are also able to affect the localization of DAF-16. In Fig. 13, a concentration dependent effect can be observed in the presence of Eleutherococcus senticosus (SHE-3). At low concentrations a translocation was only seen in a limited number of cells, whereas at higher concentrations more cells reacted and the reaction per cell 
Fig. 12 Effect of heat shock on localization of DAF16::GFP in C. elegans (TJ356 strain). Heat shock $\left(35^{\circ} \mathrm{C}\right.$ during $20 \mathrm{~min}$ ) induces a clear translocation of the DAF16 transcription factor into the nucleus
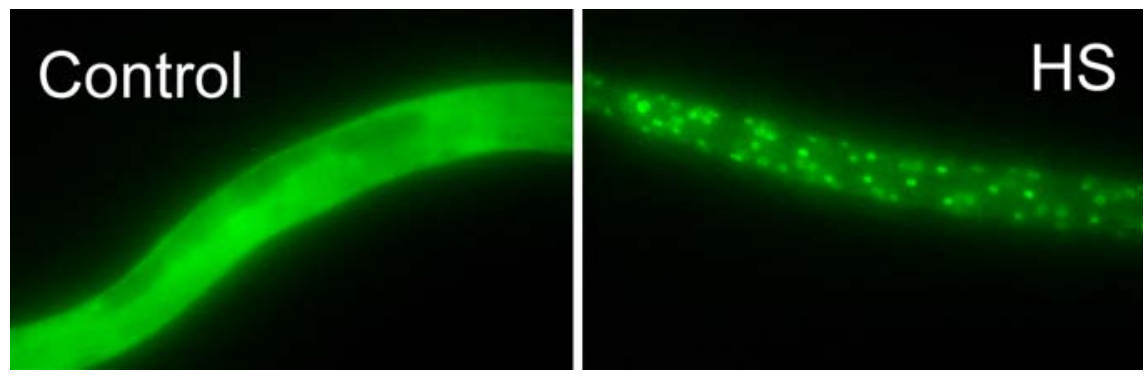

Fig. 13 Eleutherococcus senticosus (SHE-3) induces a translocation of DAF16::GFP into the nucleus in $C$. elegans (TJ356 strain). Although $100 \mu \mathrm{g} / \mathrm{ml}$ Eleutherococcus hardly induces a nuclear translocation, higher concentrations (250 and especially $500 \mu \mathrm{g} / \mathrm{ml}$ ) induce a clear translocation in most of the 50 nematodes that were observed per experimental condition. Representative examples are shown per condition
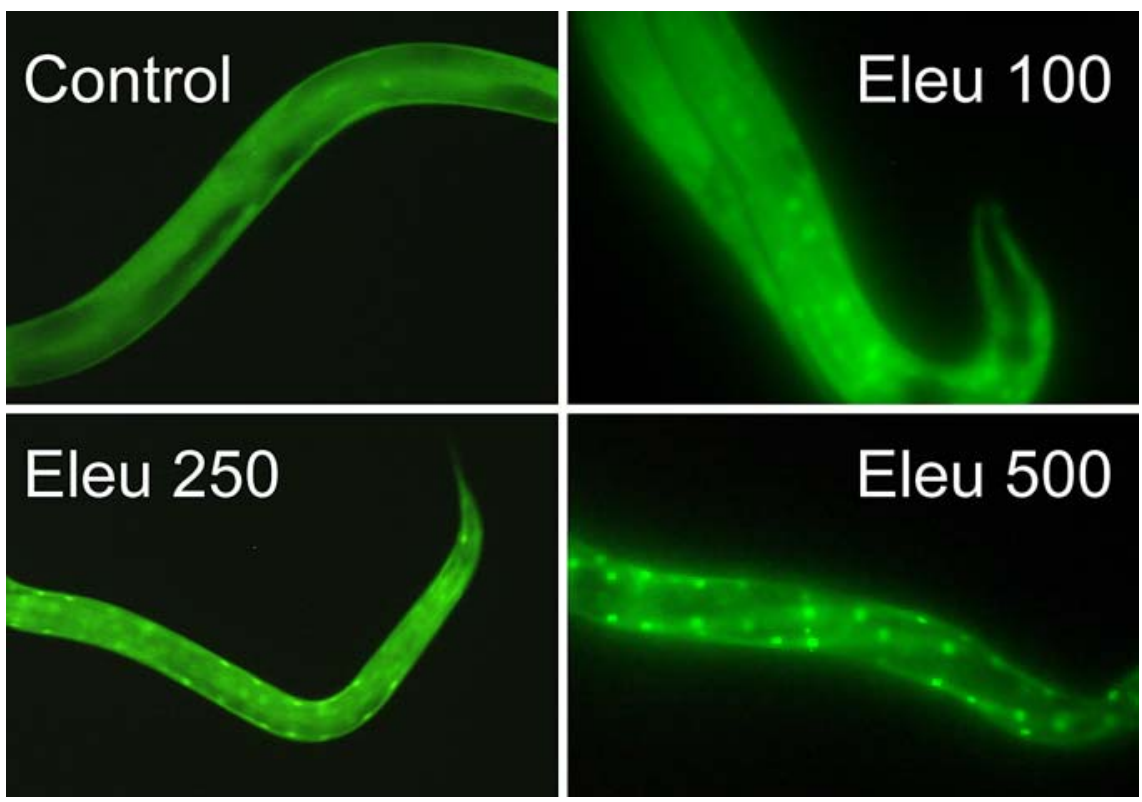

was more pronounced leading to an almost complete translocation of all DAF-16 molecules. Rhodiola rosea (SHR-5) also showed a concentration dependent effect on DAF-16 translocation to the nucleus (Fig. 14). Both extracts were thus able to induce DAF-16 translocation. However, it should be noted that the translocation following heat shock, which was used as a positive control, was often more complete in the sense that the contrast in fluorescence intensity between the nucleus and cytoplasm was more pronounced in heat shocked worms in comparison with the adaptogen-treated worms.

Do plant adaptogens increase the synthesis of HSP-16?

HSP-16 is considered to be an important chaperone protein involved in stress resistance and a strong transcriptional upregulation of HSP-16 is commonly observed in response to mild (thermal) stress, which subsequently plays a key role in the stress resistant state of the organism.

For this part of the study, the transgene strain CL2070 was used containing a construct in which the gene coding for GFP was coupled to the promoter of the hsp-16 gene (Link et al. 1999). Animals with this reporter construct do not express detectable GFP under standard conditions. However, following exposure to stress conditions, such as a heat shock $\left(35^{\circ} \mathrm{C}\right.$ for $20 \mathrm{~min}$ ), the synthesis of GFP is induced through an activation of the heat shock promoter. This transgene allows visualization of the stress response in living animals in which the amount of synthesized GFP is used as a measure of experienced stress. The presence of GFP was evaluated by processing worms for gel electrophoresis and immunoblotting using an antibody against GFP, allowing for its sensitive detection. In order to determine whether adaptogens were able to induce the synthesis of HSP-16, worms were incubated overnight on plates containing 
Fig. 14 Rhodiola rosea (SHR-5; 25-100 $\mu \mathrm{g} / \mathrm{ml}$ ) induce nuclear translocation of DAF16::GFP in C. elegans (TJ356 strain). The higher concentrations induce a more obvious translocation in most of the 50 nematodes that were observed per experimental condition. Representative examples are shown per condition
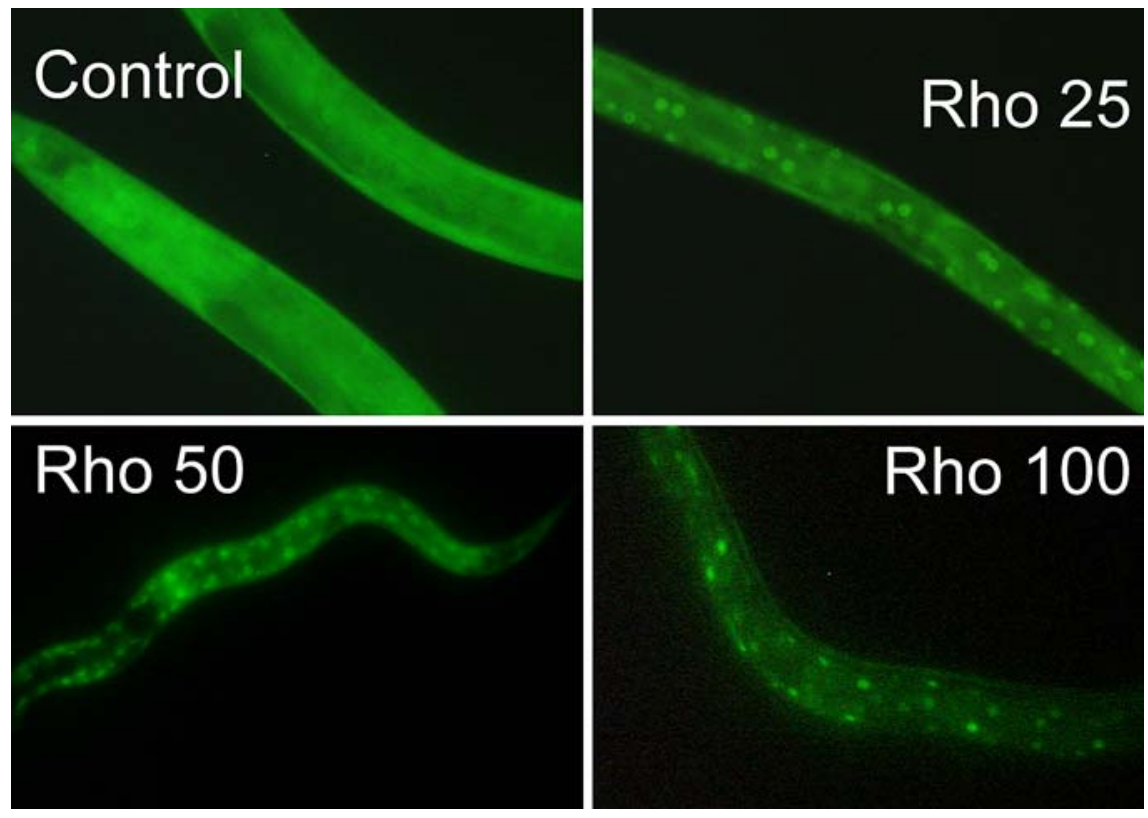

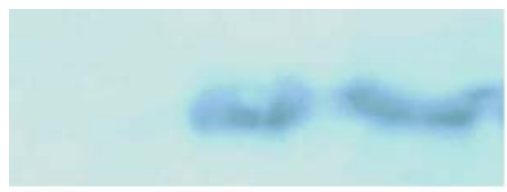

Control Eleu 250 Eleu 500

Fig. 15 Adaptogens and heat shock induce expression of GFP in the C. elegans transgene strain (CL2070), where the hsp16-2 promotor is fused to GFP. Western blot analysis showed an

different concentrations of plant adaptogen extracts. As shown in Fig. 15, both Eleutherococcus and Rhodiola were able to induce the synthesis of GFP in independent experiments, indicating their ability to activate the heat shock promoter. The induction of HSP-16 synthesis by the adaptogens was in general much weaker than the one induced by heat stress (Fig. 15) and was hardly detectable by fluorescence microscopy (not shown).

\section{Discussion}

In this paper it is shown that extracts from plant adaptogens (Eleutherococcus senticosus (SHE-3) and Rhodiola rosea (SHR-5)) are able to modulate the normal lifespan of C. elegans in a dose-dependent way. The most efficient concentrations were able to increase the mean lifespan with about $15 \%$, whereas

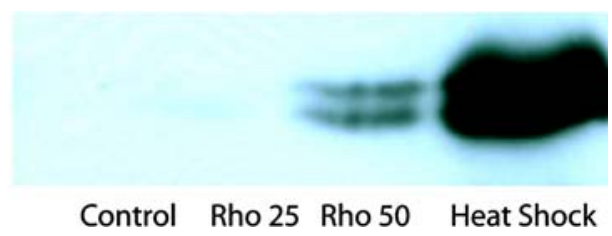

induction of GFP by the indicated concentrations of Eleutherococcus and Rhodiola as well as by a heat shock $\left(35^{\circ} \mathrm{C}\right.$ for $20 \mathrm{~min})$

higher concentrations had either no effect or even shortened the mean lifespan. Interestingly, the mean lifespan could also be increased when these adaptogens where added later in life. Apparently, it is 'never too late to start' exposing worms to adaptogens in order to increase their lifespan. In addition, an increase in stress resistance was observed when worms were incubated in the presence of adaptogens. These observations raise a number of questions which are discussed below.

Are adaptogens unique as plant products that increase the mean lifespan of $C$. elegans?

In recent studies it has been demonstrated that also other extracts or isolated compounds from plants like Ginkgo biloba (Wu et al. 2002; Kampkötter et al. 2007), blueberry extracts and its polyphenol fraction (Wilson et al. 2006) as well as resveratrol from the 
grape skin (Gruber et al. 2007) were able to increase the normal lifespan in C. elegans. In this respect, there is a lot of interest in identifying compounds that may delay the aging process for which $C$. elegans is a suitable model (Collins et al. 2006). Plant adaptogens seem to represent an additional group of plant extracts and components worthy of further analysis. Interestingly, Jafari et al. (2007) recently showed that Rhodiola rosea was able to increase the mean lifespan of Drosophila melanogaster.

How do plant adaptogens prolong lifespan?

In this paper we studied whether plant adaptogens were able to interfere with the localization of the DAF-16 transcription factor. DAF-16 is considered to be a crucial transcription factor in stress resistance and lifespan determination (Partridge and Gems 2002; McElwee et al. 2007). It was shown that adaptogens induce a nuclear localization of DAF-16. Once in the nucleus DAF-16 initiates the transcription of a large number of genes involved in metabolism and oxidative stress resistance, including chaperones and detoxifying proteins, which may explain a change in the rate of ageing and longevity (Lee et al. 2003; Murphy et al. 2003; Oh et al. 2006). Compounds that interfere with the IGF-1 signal transduction pathway and DAF-16 localization have recently been suggested as interesting candidates for pharmacological manipulation of lifespan (Gami and Wolkow 2006). With respect to the question how the localization of DAF-16 is affected, it has been shown that mild stress (including heat stress, oxidative stress and caloric restriction) induce a nuclear localization of DAF-16 (Cypser et al. 2006; Henderson and Johnson 2001; Kondo et al. 2005). This raises the intriguing question whether adaptogens exert their beneficial effect by inducing some mild stress first. In this respect it is of interest that we recently demonstrated a pro-oxidative action of the adaptogens in cells in culture (Wiegant et al. 2008). Extracts of both Eleutherococcus as well as of Rhodiola exerted a prooxidant action to which an adaptive response was generated triggering a cellular anti-oxidative defense mechanism resulting in a more stress resistant state (Wiegant et al. 2008). These results obtained in cell cultures were concentration dependent. Low doses enhanced the antioxidative defense, whereas higher doses of adaptogens were shown to cause oxidative damage leading to cell death. This supports the observations in this paper, where low concentrations cause an increase in stress resistance and mean lifespan of C. elegans, whereas the highest concentrations cause a reduction in the mean lifespan.

Caloric restriction is also known to affect DAF-16 localization and to induce lifespan prolongation. The possibility should be excluded that adaptogens may cause DAF-16 localization in an indirect way due to caloric restriction. This could be postulated in case the adaptogens kill or affect the growth of bacteria that serve as food source of the worms throughout the experiment. However, we confirmed that bacterial growth is not affected by adaptogens (not shown).

An often mentioned hypothesis is that extracts of plants, and especially their phenolic components, might also exert a beneficial lifespan enhancing effect by acting as antioxidants and thus reducing oxidative stress generated by mitochondria during metabolism. This hypothesis is in line with the 'free radical theory of aging' proposed more than 50 years ago by Harman (1956)(Kregel and Zhang (2007) for a recent overview on this theory). In this respect, the phenolic components present in plant adaptogen extracts may also exert an anti-oxidative action that could explain the observed life-span prolongation. The question is thus which supposed aspect of adaptogen action (antior pro-oxidative effect) might be most important in explaining the observed effect. It is of interest that recently both the anti-oxidative and the pro-oxidative potential of a variety of beneficial dietary compounds (including phytochemicals such as resveratrol and curcumin) have been discussed (Mattson and Cheng 2006; Mattson 2008a; Halliwell 2007; Alarcon de la Lastra and Villegas 2007) in which a beneficial effect of the pro-oxidative action of these nutritional components is usually explained in terms of an induction of anti-oxidative endogenous defense mechanisms. Further detailed research might focus on this aspect to elucidate the underlying mechanism. However, it should be noted that the experiments with the mev-1 mutant in the present paper may provide some indication. The mev-1 mutant (Ishii et al. 1998) has been used to study a possible antioxidative action of plant extracts and of specific compounds, such as vitamin $\mathrm{E}$ and Ubiquinon Q10. The latter compounds are able to quench the enhanced superoxide production in mitochondria of the mev-l mutant, and were shown to increase its shortened lifespan (Ishii et al. 
2004). Previously, an extract of Ginkgo biloba has also been shown to reduce oxidative stress in the mev-1 mutant (Wu et al. 2002). In the present study the adaptogens were only partly able to increase the lifespan of the mev-1 mutant, Eleutherococcus being more efficient than Rhodiola. This suggests that the beneficial effect of Eleutherococcus but not of Rhodiola can only partly be explained by a possible antioxidative action of the extract.

The poor beneficial effect of adaptogens in $m e v-1$ mutants might be explained by a pro-oxidative action of the extracts studied. A pro-oxidative action may be of relevance when adaptogens in low doses increase endogenous defense and lead to life span prolongation. However, an organism which already suffers from an increased level of reactive oxygen species will probably not benefit from an additional prooxidative action. On the contrary, the concentrations that are beneficial under normal conditions might now be experienced as detrimental, which is in agreement with our observations.

If adaptogens induce their effect as mild stressors, it is of interest to compare them with hormetic components, since the initial beneficial effect and subsequent adverse effect of both adaptogens suggests and resembles a hormetic action.

Do adaptogens resemble hormetic components in their mild stress action?

In this paper we observed that low concentrations of adaptogens cause an increase in stress resistance and mean lifespan of $C$. elegans, whereas the highest concentrations cause a reduction in the mean lifespan. Similar findings have been described in the field of longevity hormesis (Neafsey 1990; Calabrese and Blain 2005; Mattson 2008b). In this respect, it is of interest that scientists from different fields recently supported more coherence in the terminology to indicate an evolutionary conserved process, where low doses of stressful stimuli activate an adaptive response aimed at increasing the resistance of a cell or organism (Calabrese et al. 2007). The effect of plant adaptogens appears to be in agreement with these observations. By acting as a mild stressstimulus it can be hypothesized that adaptogens activate an adaptive response leading to an increase in resistance of the organism to a subsequent stress and at the same time by increasing maintenance, repair and resistance processes via the IGF-1 pathway and via DAF-16 localization, they also support an increase in lifespan. In this sense, it can be suggested that adaptogens may act as hormetic agents (or 'hormetins'). Such a mechanism of action is in agreement with current anti-aging strategies in the field of hormesis (Rattan and Clark 2005; Rattan 2008). With the aim to design anti-aging strategies a variety of mild stressful stimuli have been recognized as interesting possibilities to activate the adaptive response and thereby increase cellular resistance and maintenance processes (Rattan 2004, 2008; Marini et al. 2008). These mild stressful stimuli include the pro-oxidant stress exerted by exercise, which raise antioxidative defense mechanisms (Banerjee et al. 2003; Li et al. 2006), repeated mild heat shock treatment (Rattan 2008), as well as various phytochemicals (Mattson and Cheng 2006; Mattson 2008a). In this respect, Mattson and Cheng (2006) and Mattson (2008a) described a number of examples of phytochemicals, which at high doses are toxic (coumarin, resveratrol, etc.) but at lower doses have a beneficial adaptive effect in the nervous system against a variety of stress conditions.

It would be of interest to compare the effect of adaptogens and the effect of known hormetic components in more detail, since there seems to be some interesting similarities. However, more detailed comparison of effects at the cellular and organism level are required before adaptogens can considered to be hormetins.

In case adaptogens exert their lifespan enhancing through stress responsive mechanisms, it could be of concern that secondary physiological mechanisms are affected. In this respect it is of interest that Jafari et al. (2007) recently showed that the lifespan enhancing effect of Rhodiola in Drosophila melanogaster was not associated with statistically significant reductions in fecundity, metabolic rate or male mating success. Using detailed analysis of their data they were able to exclude for instance that the effects of Rhodiola could be explained as a caloric-restriction effect. As mentioned before, we also excluded the possibility that adaptogens prevented the growth of bacteria that serve as food source for the worms throughout the life span experiments. Growth of bacteria was not affected by adaptogens and thus it could be excluded that adaptogens might indirectly induce lifespan prolongation by way of caloric restriction. 
Currently, compounds in the extracts are being studied, which can be held responsible for the lifespan prolonging effect described in the present study. In this respect a number of major compounds in extracts from plant adaptogens have been identified, isolated and characterized, such as Eleutherosides in Eleutherococcus and some major components in Rhodiola such as salidroside, rosavin, and tyrosol amongst others. Apart from a search for responsible single components, a parallel approach aimed at identifying synergy between single compounds is of interest (Wiegant and Surinova 2006).

\section{Conclusion}

In the present paper, it is shown that the plant adaptogens Eleutherococcus senticosus (SHE-3) and Rhodiola rosea (SHR-5) are able to increase the lifespan of $C$. elegans dose-dependently.

The most effective beneficial concentrations of Eleutherococcus $(250 \mu \mathrm{g} / \mathrm{ml})$ and of Rhodiola $(10-25 \mu \mathrm{g} / \mathrm{ml})$ did not only extend the mean lifespan with about 2 days (15\%), but were also able to increase the maximum lifespan with about 3 days.

In addition, adaptogens increased the resistance of C. elegans to a number of stress conditions such as heat stress, UV exposure and oxidative stress. Finally, it was demonstrated that adaptogens induced a translocation of the transcription factor DAF-16 into the nucleus. This observation at the molecular level might explain a shift towards processes involved in maintenance, repair and stress resistance due to a change in gene expression.

Acknowledgement Caenorhabditis elegans strains were provided by the Caenorhabditis Genetics Center, at the University of Minnesota, which is funded by the NIH National Center for Research Resources (NCRR).

Open Access This article is distributed under the terms of the Creative Commons Attribution Noncommercial License which permits any noncommercial use, distribution, and reproduction in any medium, provided the original author(s) and source are credited.

\section{References}

Afanasiev SA, Krylatov AV, Lasukova TV, Lishmanov YB (1996) The role of inducible stress proteins in cardioprotective effects of Rhodiola rosea. J Biokhim 61:1779-1784
Alarcon de la Lastra C, Villegas I (2007) Resveratrol as an antioxidant and pro-oxidant agent: mechanisms and clinical implications. Biochem Soc Trans 35(5):1156-1160. doi:10.1042/BST0351156

Banerjee AK, Mandal A, Chanda D, Chakrabort S (2003) Oxidant, antioxidant and physical exercise. Mol Cell Biochem 253:307-312. doi:10.1023/A:1026032404105

Boon-Niermeijer EK, van den Berg A, Wikman G, Wiegant FAC (2000) Phyto-adaptogens protect against environmental stress-induced death of embryos from the freshwater snail Lymnaea stagnalis. Phytomedicine 7:389-399

Brekhman II (1980) Man and biologically active substance: the effect of drugs, diet and pollution on health. Pergamon Press, Oxford

Brekhman II, Dardymov IV (1969) New substances of plant origin which increase non-specific resistance. Annu Rev Pharmacol 9:419-430. doi:10.1146/annurev.pa.09.040 169.002223

Brenner S (1974) The genetics of Caenorhabditis elegans. Genetics 77:71-94

Brown RP, Gerbarg PL, Ramazanov Z (2002) Rhodiola rosea; a phytomedicinal overview. HerbalGram 56:40-52. (www. herbalgram.org)

Calabrese EJ, Blain R (2005) The occurrence of hormetic dose responses in the toxicological literature, the hormesis database: an overview. Toxicol Appl Pharmacol 202:289301. doi:10.1016/j.taap. 2004.06.023

Calabrese EJ et al (2007) Biological stress response terminology: integrating the concepts of adaptive response and preconditioning stress within a hormetic dose-response framework. Toxicol Appl Pharmacol 222(1):122-128. doi:10.1016/j.taap. 2007.02.015

Chiu PY, Tang MH, Mak DHF, Poon KT, Ko KM (2003) Hepatoprotective mechanism of schisandrin $\mathrm{B}$ : role of mitochondrial glutathione antioxidant status and heat shock proteins. Free Radic Biol Med 35:368-380. doi: 10.1016/S0891-5849(03)00274-0

Collins JJ, Evason K, Kornfeld K (2006) Pharmacology of delayed aging and extended lifespan of Caenorhabditis elegans. Exp Gerontol 41:1032-1039. doi:10.1016/ j.exger.2006.06.038

Cypser JR, Tedesco P, Johnson TE (2006) Hormesis and aging in Caenorhabditis elegans. Exp Gerontol 41:935-939. doi: 10.1016/j.exger.2006.09.004

Davydov M, Krikorian AD (2000) Eleutherococcus senticosus (Rupr. \& Maxim.) Maxim. (Araliacea) as an adaptogen: a closer look. J Ethnopharmacol 72:345-393. doi: 10.1016/S0378-8741(00)00181-1

De Sanctis R, De Bellis R, Scesa C, Mancini U, Cuchiarini L, Dacha M (2004) In vitro protective effect of Rhodiola rosea extract against hypochlorous acid-induced oxidative damage in human erythrocytes. Biofactors 20: 147-159

Gami MS, Wolkow CA (2006) Studies of Caenorhabditis elegans DAF-2/insulin signaling reveal targets for pharmacological manipulation of lifespan. Aging Cell 5:3137. doi:10.1111/j.1474-9726.2006.00188.x

Gruber J, Tang SY, Halliwell B (2007) Evidence for a trade-off between survival and fitness caused by resveratrol treatment of Caenorhabditis elegans. Ann NY Acad Sci 1100:530-542. doi:10.1196/annals.1395.059 
Guarente L, Kenyon C (2000) Genetic pathways that regulate ageing in model organisms. Nature 408:255-262. doi: $10.1038 / 35041700$

Halliwell B (2007) Dietary polyphenols: good, bad, or indifferent for your health? Cardiovasc Res 73:341-347. doi: 10.1016/j.cardiores.2006.10.004

Harman D (1956) Aging, a theory based on free radical and radiation chemistry. J Gerontol 11:298-300

Henderson ST, Johnson TE (2001) daf-16 integrates developmental and environmental inputs to mediate aging in the nematode Caenorhabditis elegans. Curr Biol 11:19751980. doi:10.1016/S0960-9822(01)00594-2

Hosono R (1978) Sterilization and growth inhibition of Caenorhabditis elegans by 5-fluorodeoxyuridine (FdUrd). Exp Gerontol 13:369-374. doi:10.1016/0531-5565(78) 90047-5

Houthoofd K, Vanfleteren JR (2007) Public and private mechanisms of life extension in Caenorhabditis elegans. Mol Genet Genomics 277:601-617. doi:10.1007/s00438-0070225-1

Ishii N, Fujii M, Hartman PS, Tsuda M, Yasuda K, SenooMatsuda N et al (1998) A mutation in succinate dehydrogenase cytochrome $b$ causes oxidative stress and ageing in nematodes. Nature 394:694-697. doi:10.1038/29331

Ishii N, Senoo-Matsuda N, Miyake K, Yasuda K, Ishii T, Hartman PS et al (2004) Coenzyme Q10 can prolong C. elegans lifespan by lowering oxidative stress. Mech Ageing Dev 125:41-46. doi:10.1016/j.mad.2003.10.002

Jafari M, Jeffrey S, Felgner JS, Bussel II, Hutchili T, Khodayari B et al (2007) Rhodiola: a promising anti-aging Chinese herb. Rejuvenation Res 10(4):587-602. doi: 10.1089/rej.2007.0560

Johnson TE, Lithgow GJ, Murakami S (1996) Hypothesis: interventions that increase the response to stress offer the potential for effective life prolongation and increased health. J Gerontol Biol Sci 51A:B392-B395

Kampkötter A, Pielarski T, Rohrig R, Timpel C, Chovolou Y, Watjen W et al (2007) The Ginkgo biloba extract EGb761 reduces stress sensitivity, ROS accumulation and expression of catalase and glutathione S-transferase 4 in Caenorhabditis elegans. Pharmacol Res 55:139-147. doi: 10.1016/j.phrs.2006.11.006

Kapahi B, Boulton ME, Kirkwood TB (1999) Positive correlation between mammalian lifespan and cellular resistance to stress. Free Radic Biol Med 26:495-500. doi: 10.1016/S0891-5849(98)00323-2

Khanum F, Bawa SB, Singh B (2005) Rhodiola rosea: a versatile adaptogen. Compr Rev Food Sci Food Saf 4:55-62. doi:10.1111/j.1541-4337.2005.tb00073.x

Kondo M, Senoo-Matsuda N, Yanase S, Ishii T, Hartman PS, Ishii N (2005) Effect of oxidative stress on translocation of DAF-16 in oxygen-sensitive mutants, mev-1 and gas-1 of Caenorhabditis elegans. Mech Ageing Dev 126:637641. doi:10.1016/j.mad.2004.11.011

Kregel KC, Zhang HJ (2007) An integrated view of oxidative stress in aging: basic mechanisms, functional effects, and pathological considerations. Am J Physiol 292: R18-R36

Lee SS, Kennedy S, Tolonen AC, Ruvkun G (2003) DAF-16 target genes that control C. elegans life-span and metabolism. Science 300:644-647. doi:10.1126/science.1083614
Li JJ, Gomez-Cabrera MC, Vina J (2006) Exercise and hormesis: activation of cellular antioxidant signaling pathways. Ann NY Acad Sci 1067:425-435

Link CD, Cypser JR, Johnson CJ, Johnson TE (1999) Direct observation of stress response in Caenorhabditis elegans using a reporter transgene. Cell Stress Chaperones 4:235242. doi:10.1379/1466-1268(1999)004 $\leq 0235$ :DOOSRI $\geq$ 2.3.CO;2

Lithgow GJ, Walker GA (2002) Stress resistance as a determinate of C. elegans lifespan. Mech Ageing Dev 123:765-771. doi:10.1016/S0047-6374(01)00422-5

Lithgow GJ, White TM, Melov S, Johnson TE (1995) Thermotolerance and extended life-span conferred by single-gene mutations and induced by thermal stress. Proc Natl Acad Sci USA 92:7540-7544. doi:10.1073/pnas.92.16.7540

Marini AM, Jiang H, Pan H, Wu X, Lipsky RH (2008) Hormesis: a promising strategy to sustain endogenous neuronal survival pathways against neurodegenerative disorders. Ageing Res Rev 7:21-33

Mattson MP (2008a) Dietary factors, hormesis and health. Ageing Res Rev 7:43-48. doi:10.1016/j.arr.2007.08.004

Mattson MP (2008b) Hormesis defined. Ageing Res Rev 7:1-7. doi:10.1016/j.arr.2007.08.007

Mattson MP, Cheng A (2006) Neurohormetic phytochemicals: low-dose toxins that induce adaptive neuronal stress responses. Trends Neurosci 29(11):632-639. doi: 10.1016/j.tins.2006.09.001

McElwee JJ, Schuster E, Blanc E, Piper MD, Thomas JH, Patel DS et al (2007) Evolutionarily conservation of regulated longevity assurance mechanisms. Genome Biol 8(7):R132. Epub ahead of print. doi:10.1186/gb-2007-8-7-r132

Meerson F (1984) Adaptation, stress and prophylaxis. Springer Verlag, Heidelberg

Muñoz MJ, Riddle DL (2003) Positive selection of Caenorhabditis elegans mutants with increased stress resistance and longevity. Genetics 163:171-180

Murphy GT, McCarroll SA, Bargmann CI, Fraser A, Kamath RS, Ahringer J et al (2003) Genes that act downstream of DAF-16 to influence the lifespan of Caenorhabditis elegans. Nature 424:277-284. doi:10.1038/nature01789

Neafsey PJ (1990) Longevity hormesis: a review. Mech Ageing Dev 51:1-31. doi:10.1016/0047-6374(90)90158-C

Oh SW, Mukhopadhyay A, Dixit BI, Raha T, Green MRR, Tissenbaum HA (2006) Identification of direct DAF-16 targets controlling longevity, metabolism and diapause by chromatin immunoprecipitation. Nat Genet 28:251-257

Panossian A, Wagner H (2005) Stimulating effect of adaptogens: an overview with particular reference to their efficacy following single dose administration. Phytother Res 19:819-838. doi:10.1002/ptr.1751

Partridge L, Gems D (2002) Mechanisms of ageing; public or private? Nat Rev Genet 3:165-175. doi: $10.1038 / \operatorname{nrg} 753$

Rattan SI (2004) Hormetic mechanisms of anti-aging and rejuvenating effects of repeated mild heat stress on human fibroblasts in vitro. Rejuvenation Res 7(1):40-48. doi: $10.1089 / 154916804323105071$

Rattan SI (2008) Hormesis in aging. Ageing Res Rev 7:63-78. doi:10.1016/j.arr.2007.03.002

Rattan SI, Clark BF (2005) Understanding and modulating ageing. IUBMB Life 57:297-304. doi:10.1080/15216540 500092195 
Stiernagle T (1999) Maintenance of C. elegans. In: Hope IA (ed) C. elegans; a practical approach. Oxford University Press, pp 51-67

Sulston JE, Brenner S (1974) The DNA of Caenorhabditis elegans. Genetics 77:95-105

Wagner H, Nörr H, Winterhoff H (1994) Plant adaptogens. Phytomedicine 1:63-76

Wiegant FAC, Surinova S (2006) Synergie: achtergrond, concepten en definities. Ned Tijdschr Fytotherap 19(3):3-10

Wiegant FAC, Limandjaja G, de Poot SAH, Bayda LA, Vorontsova ON, Zenina TA et al (2008) Plant adaptogens activate cellular adaptive mechanisms by causing mild damage. In: Lukyanova L, Takeda N, Singal PK (eds) Adaptation biology and medicine: health potentials, vol 5 . Narosa Publishers, New Delhi, India, pp 319-332

Wilson MA, Shukit-Hale B, Kalt W, Ingram DK, Joseph JA, Wolkow CA (2006) Blueberry polyphenols increase lifespan and thermotolerance in Caenorhabditis elegans. Aging Cell 5:59-68. doi:10.1111/j.1474-9726.2006. 00192.x

Wu Z, Smith JV, Paramasivam V, Butko P, Khan I, Cypser JR et al (2002) Ginkgo biloba extract EGb 761 increases stress resistance and extends life span of Caenorhabditis elegans. Cell Mol Biol 48:725-731 